\title{
ПРИМЕНЕНИЕ НЕЙРОННОЙ СЕТИ “НЕОКОГНИТРОН" ДЛЯ ОБРАБОТКИ ИЗОБРАЖЕНИЙ ИНТЕГРАЛЬНЫХ СХЕМ
}

\author{
М.E. Ваткин ${ }^{1)}$, Р.X. Садыхов ${ }^{2)}$
}

1) Институт технической кибернетики НАН Беларуси, 220012, Беларусь, г. Минск, ул. Сурганова 6, vatkin@tut.by 2)Беларуский государственный университет информатики и радиоэлектроники, 220600, Беларусь, г. Минск, ул. Петруся-Бровки 6, тел./фракс. (017)2310982, rsadykhov@gw.bsuir.unibel.by

Аннотация. Рассматрив аются архитектура нейронной сети “неокогнитрон” в задачах поиска структурных элементов на полутоновых изображениях интегральных схем. Предлагается модифицированное правило активачии нейронов инвариантное к искажениям яркости изображения. Сравнительные результаты применения нейронной сети показали преимущество использования нейросетевого подхода.

Ключевые слова: Нейронная сеть, Распознавание образов

\section{1. ВВЕДЕНИЕ}

В настоящее время применяются различные виды нейронных сетей в задачах распознавания и анализа изображений.[1-4]. В статье представлена технология поиска структурных элементов на полутоновых изображениях интегральных микросхем (ИМС) основанная на применении многослойной нейронной сети. Алгоритм поиска отдельных структурных единиц на изображении ИМС реализуется методом сканирования этого изображения скользящим окном для каждой позиции которого определяется мера соответствия изображения попадающего в окно с искомым эталонным изображением. Для определения меры соответствия была разработана нейронная сеть «неокогнитрон» с упрощенной структурой и адаптированным алгоритмом ее обучения.

\section{2. СТРУКТУРА НЕЙРОННОЙ СЕТИ}

Обобщенная структура нейронной сети представлена на рис 1.

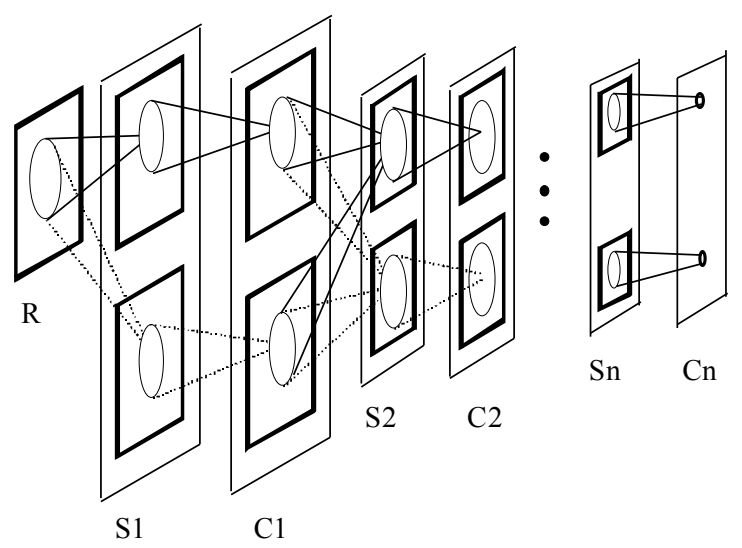

Рис.1 Структура нейронной сети
В структуре нейрокогнитрона выделяют следующие элементы: $R$-слой, $S$-слои, $C$-слои, $\bar{S}$-подслои, $\bar{C}$-подслои, $\widetilde{S}$-нейроны, $\widetilde{C}$ нейроны, $\breve{S}$-связи, $\breve{C}$-связи. $R$-слой является рецепторным слоем который преобразует входное изображение в значения активностей его пикселей передаваемые далее по сети. $S$ и $C$-слои изображены на рисунке тонкой линией, они состоят $\widetilde{S}$ и $\widetilde{C}$-нейронов, которые осуществляют обнаружение на изображении различных его характеристик, таких как линии, их пересечения, углы и т.д. Каждый $S$ и $C$-слойг делится на $\bar{S}$ и $\bar{C}$-подслои, которые изображены жирной линией. Таким образом, $\bar{S}$ подслой формирует карту распределения признака в предыдущем слое.

$\widetilde{S}$-нейроны имеют входящие $\breve{S}$-связи, по которым в нейрон поступают значения активности нейронов от предыдущего слоя. Каждая группа связей образует свое рецепторное поле, которое характеризуется такими параметрами как позиция и размер. Размер каждого рецепторного поля определяется размерами характеристики изображения которую данная группа связей передает для идентификации $\widetilde{S}$-нейрону.

Позиция групп рецепторных полей определяется позицией соответствующего им $\widetilde{S}$ нейрона. Таким образом рецепторные поля $\widetilde{S}$ - нейронов из одного $\widetilde{S}$ - подслоя имеют равные размеры, а их позиции отличаются параллельным сдвигом относительно друг друга. Т.к. нейроны из одного $\widetilde{S}$ - подслоя 
обнаруживают одинаковые характеристики изображения отличающиеся только позицией, то обучается только один $\widetilde{S}$ - нейрон, а значения весовых коэффициентов остальных нейронов из этого подслоя приравниваются значениям весовых коэффициентов обученного нейрона.

$\widetilde{S}$-нейрон обучается с помощью функции изменения весовых коэффициентов:

$$
w(t+1)=w(t)+\frac{1}{t+1} \cdot[u(t+1)-w(t)]
$$

где $t$ - номер обучающей итерации, $w$ - значение весового коэффициента, $u$ - значение активности нейрона из рецепторного поля к которому присоединена данная связь.

Функция активации $\widetilde{S}$-нейрона соответствует радиальной базисной функции:

$$
\left.U_{S l}(n, k)=\exp \sqrt{\frac{\sum_{p=1}^{P_{C L}} \sum_{v \in A l}\left(\widetilde{U}_{C l}(n+v, p)-n(v, k, p)\right)^{2}}{N}}\right)
$$

где $l$ - номер слоя; $k$ - номер обучаемого подслоя; $n$ - двумерный индекс нейрона в $k$-й подслое; $w$ - весовой коэффициент связи; $v$ двумерный индекс связи в $p$-й группе связей; $A l$ - двумерное значение соответствующее размеру рецепторного поля в предыдущем подслое, $N$ - общее количество нейронов в рецепторном поле нейрона, $p$ - номер группы связей. Аргумент

$$
\widetilde{U}_{C l}(n+v, p)=U_{C l}(n+v, p)-\min _{\forall v}\left(U_{C l}(n+v, p)\right)
$$

введен в соотношение (2) с целью исключения влияния константной компоненты яркости изображения. Это обеспечивает инвариантность сети к изменениям яркости распознаваемого изображения.

$\bar{C}$-подслой состоит из $\widetilde{C}$-нейронов, которые обобщают характеристики изображения обнаруженные в предыдущем слое. Обобщение означает, что если в рецепторное поле $\bar{C}$ нейрона попадает хотя бы один активный нейрон, то $\bar{C}$ - нейрона тоже переходит в активное состояние. Т.е. для всех значений активностей из рецепторного поля применяется функция «ИЛИ» нечеткой логики, а ее результат отождествляется с выходной активностью $\bar{C}$ - нейрона.

$U_{C l}(n, k)=\max \left(U_{S l-1}(n+v, p)\right), \forall v \in D l, \forall p \in P$

где $l$ - номер слоя; $p, P$ - номер слоя и множество подслоев из рецепторного поля $\widetilde{C}$ нейрона; $D l$ - двумерное значение соответствующие размерам рецепторных полей $\widetilde{C}$ нейрона; $v$ - двумерный индекс связи внутри каждой группы связей.

Обычно $\widetilde{C}$-нейроны образуют рецепторные поля в одном подслое предыдущего слоя, или в нескольких, когда необходимо комбинировать характеристики изображения обнаруженные в предыдущем слое. Например, когда в $\bar{C}$ подслое необходимо выделять перепад яркостей, тогда рецепторные поля связей находятся в двух $\bar{S}$-подслоях, первый из которых определяет перепад с яркого на темный, а другой с темного на яркий.

Позиция рецепторных полей $\widetilde{C}$-нейрона определяются также как для $\widetilde{S}$-нейрона.

\section{3. ОБУЧЕНИЕ}

Примененная структура нейронной сети изображена на рис. 2 , где $R$-слой рецепторный слой. Размер рецепторного слоя равен размеру искомого структурного элемента ИМС.

$S 1$-слой предназначен для обнаружения общих для всех ИМС таких, как линии различных ориентаций. Все подслои этого слоя состоят из нейронов с одинаковыми размерами рецепторных полей равными 4x4 нейронов. Обучающие изображения для этих подслоев изображены на рис. 3.

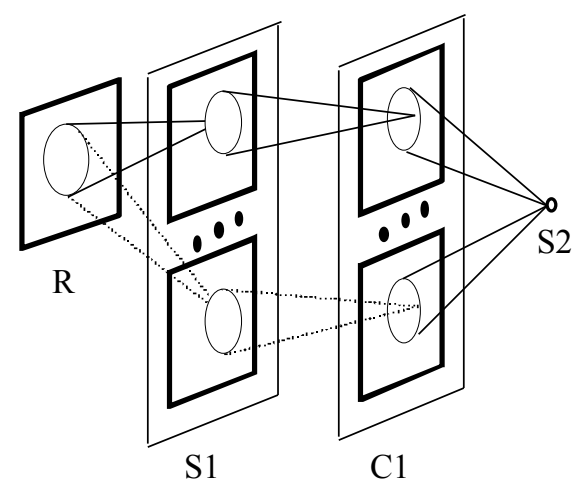

Fig.2 Использованная структура сети 
C1-слой предназначен для обобщения характеристик изображения обнаруженных в слое $S 1$-слое, а группы связей нейронов организованы так, чтобы объединять такие пары характеристик, как перепады яркостей различной ориентации. На рис. 3 такие признаки объединены скобкой. В результате получаем в $C 1$-слое 4 подслоя. Размер рецепторного полей таковы, чтобы активность нейронов и этого слоя была инвариантна к малым сдвигам характеристик изображения и составляет поле $2 \times 2$ нейрона.

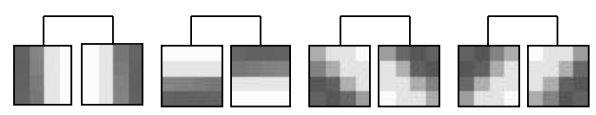

\section{Рис.3 Обучающие изображения S1-слоя}

$S 2$-слой предназначен для обнаружения характеристик изображения принадлежащих отдельному элементу ИМС. Поскольку $S 2$-слой формирует выходное значение сети, то он состоит из одного $\widetilde{S}$-нейрона, а размеры рецепторных полей нейрона совпадают с размерами $\bar{C}$-подслоев из предыдущего слоя. Т.к. весовые коэффициенты этого нейрона содержат уникальную информацию об искомом элементе, то возможно создание базы данных искомых элементов ИМС.

Структура программной системы представлена на рис. 4

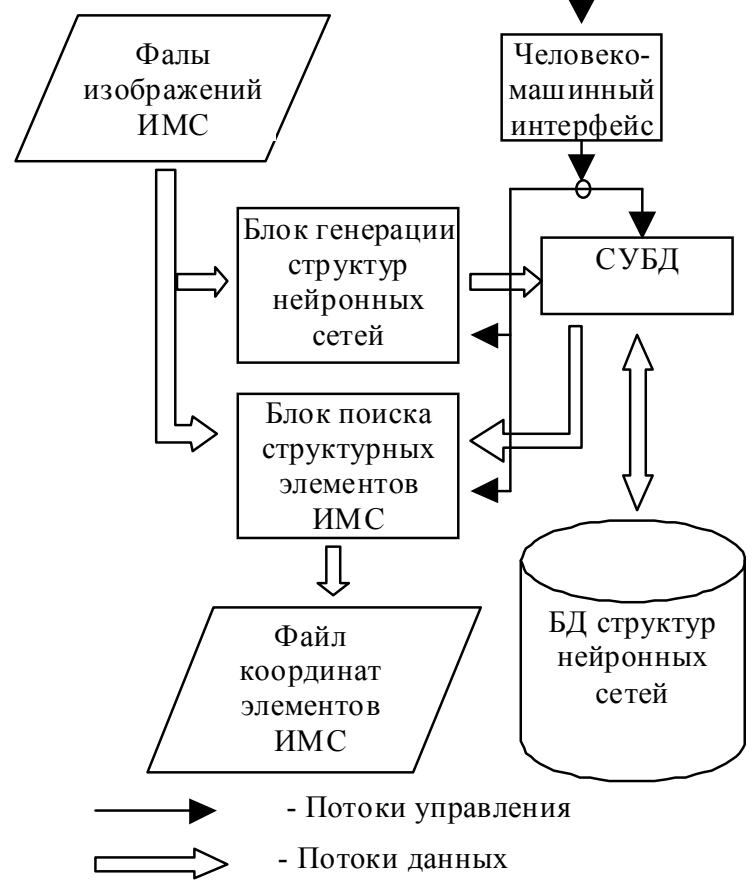

Рис.4 Структура программной сист емы

Принимая во внимание описанный выше подход к генерации структуры нейронной сети, изображенная на рисунке программная система выполняет следующую очередность действий:

1. Запрашиваются у пользователя тренировочные изображения или загружается из БД подходящая структура обученной нейронной сети

2. По обучающим образам обучается нейронная сеть.

3. Весовые коэффициенты обученной нейронной сети, а также размер рецепторного слоя сохраняются в БД.

4. С помощью нейронной сети выполняется поиск требуемого элемента на изображении ИМС, а результаты поиска записываются в выходной файл в виде пар координат положения искомого элемента.

\section{4. ТЕСТИРОВАНИЕ}

В качестве входных тестовых данных использовались полутоновые фотоизображения полиселиконного слоя ИМС (рис.5).

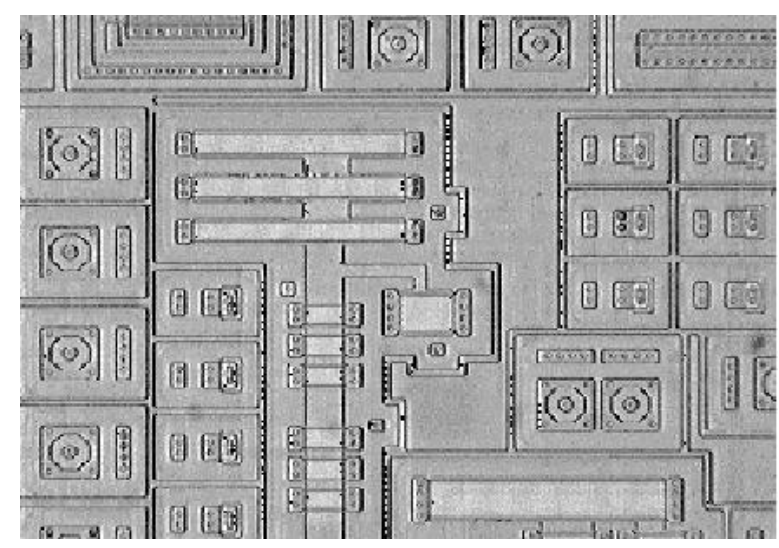

Рис.5 Изображения полиселиконого слоя ИМС

Производилось два варианта обучения нейронной сети. Обучающие образы для этих вариантов изображены на рис. 6

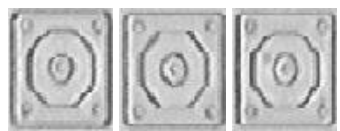

Вариант 1

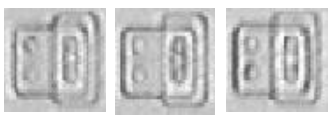

Вариант 2

\section{Рис.6 Обучающие изабражения для $\boldsymbol{S 2 - с л о я ~}$}

Для определения эффективности нейронной сети в качестве меры соответствия данный подход сравнивался с методом расчета коэффициента корреляции. Коэффициент корреляции рассчитывается между изображением получаемым из окна скользящего по ИМС и изображением усредненным по всем обучающим изображениям. Коэффициент корреляции вычисляется следующим образом: 


$$
\mu=\frac{\sum_{x, y} a_{x, y} \cdot b_{x, y}}{\sqrt{\sum_{x, y} a_{x, y}^{2} \cdot \sum_{x, y} b_{x, y}^{2}}}
$$

и увеличения точности распознавания. Средняя точность распознавания составила $98,7 \%$.

где $x, y$ - координаты точек изображения которые будут сравниваться, $a, b$ - значения цвета точки для усредненного изображения и для изображения из скользящего окна.

Результаты тестирования можно наблюдать в таблице 1. Тестирование показало, что нейросетевой подходпоказал лучший результат по отношению к корреляционному методу. Этот результат получен т.к. нейронная сеть инвариантна по отношениям к искажениям формы и яркости распознаваемого изображения, а также требует меньшего количества обучающих изображений.

Таблица 1. Результаты тестирования

\begin{tabular}{|c|c|c|c|c|}
\hline \multirow{2}{*}{$\begin{array}{c}\text { Размер } \\
\text { обучаю- } \\
\text { щего }\end{array}$} & \multicolumn{2}{|c|}{ Точность (процент распознавания) } \\
\cline { 2 - 5 } $\begin{array}{c}\text { мнтод } \\
\text { моролес- } \\
\text { тва }\end{array}$ & Variant 1 & \multicolumn{2}{|c|}{ Нейрониая сеть } \\
\hline 1 & 63,1 & 67,4 & 87,0 & 89,0 \\
3 & 72,3 & 73,6 & 96,3 & 97,0 \\
6 & 81,4 & 84,2 & 98,5 & 99,0 \\
\hline
\end{tabular}

\section{5. ЗАКЛЮЧЕНИЕ}

Представлен алгоритм обработки полутоновых изображений основанный на нейронной сети «неокогнитрон». Разработанный алгоритм активации нейронов инвариантен по отношению к асцеляциям яркости распознаваемого изображения. Показано преимущество нейросетевого подхода при распознавании изображений по отношению к методу корреляции в рамках уменьшения размеров обучающего множества

\section{6. ЛИТЕРАТУРА}

[1] G. A. Carpenter, W. D. Ross. ART-EIMAGE: $A$ neural network architecture for object recognition by evidence accumulation, IEEE Transaction on Neural Networks 6 (1995). p. 805-818.

[2] G. A. Carpenter, S. Grossberg, G. W. Lesher. The What-and-Were Filter. A Spatial Imageping Neural Network for Object Recognition and Image Understanding, Computer Vision and Image Understanding 69 (1) (1998). p. 1-22.

[3] S. Grossberg, E. Mingolla, J. Williamson. Synthetic aperture radar processing by multiple scale neural system for boundary and surface representation, Neural Networks 8 (1995). $p$. 1005-1028.

[4] G.H. Traven. A Neural Network Approach to Statistical Pattern Classification by "Semiparametric" Estimation of Probability Density Function, IEEE Transactions on Neural Networks 2 (3) (1991). p. 366-377.

[5] K. Fukushima. Neocognitron: A self-organizing neural network model for a mechanism of pattern recognition unaffected by shift in position, Biol. Cybern. 36 (1980). p. 193-202.

[6] K. Fukushima, S. Miyake. Neocognitron: A new algorithm for pattern recognition tolerant of deformations and shifts in position, Pattern Recognition 15 (1982). p. 455-469.

[7] K. Fukushima, S. Miyake, T. Ito. Neocognitron: A neural network model for a mechanism of visual pattern recognition, IEEE Trans. Syst., Man, Cybern. SMC-13 (1983). p. 826-834.

[8] K. Fukushima, N. Wake. Handwritten alphanumeric character recognition by the Neocognitron, IEEE Trans. on Neural Networks 2 (3) (1991). p. 355-365.

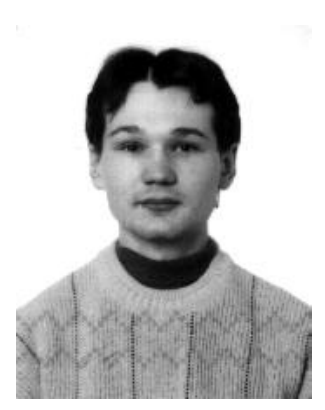

Ваткин Максим Евгеньевич в 1998 окончил $C$ отличием Брестский политехнический институт по специальности «инженер системотехник». С 1998 по 2001 обучался в аспирантуре Института технической кибернетики г. Минска. В настоящее время являюсь младшим научным сотрудником в лаборатории “Идентификации систем" Института технической кибернетики НАН Беларуси.

Садыхов Рауф Хосровович окончил в 1967 году Азербайджанский политехнический институт (Баку) по специальности "Математические и счетно-решающие приборы и устройства".
После окончания института поступил в аспирантуру в Институт Технической Кибернетики 2. Минск. В 1991 защитил диссертацию на степень доктора технических наук $в$ области кибернетики и в 1992 получил звание профрессора. C 1995 Садыхов P. Х. является заведующим кафредрой ЭВМ в Белорусском государственном университете информатики и радиоэлектроники и одновременно заведующим лаборатории Идентифиикации Систем в Институте технической кибернетики НАН Беларуси. 